Kapitalfreiheit in Europa berufen. Einer gesetzgeberischen Zielquote stehen sie nicht entgegen, wie bereits Windbichler in ihrem Gutachten vom Mai 2008 anlässlich einer Anhörung im Deutschen Bundestag zu einem Antrag der Fraktion der Grünen zu Frauenquoten für Aufsichtsräte börsennotierter Unternehmen deutlich gemacht hat. Sie hat in dieser in dieser Stellungnahme keineswegs ein verfassungs- bzw. europarechtliches Verdikt für Zielquoten ausgesprochen. ${ }^{21}$ Aus heutiger Sicht werden darüber hinaus bei der Auslegung der „Freiheiten “ die Wertsetzungen des Lissabon-Vertrages berücksichtigt werden müssen, wie sie in Art. 2 und 3 des EUV, sowie Art. 8 AEUV ihren Niederschlag gefunden haben:

Art. 2 des EUV stellt klar, dass die Gleichheit von Frauen und Männern ein Wert der Union ist. Art. 3 Abs. 3 des EUV stellt in S. 3 klar, dass die EU proaktiv für die Gleichstellung von Frauen und Männern eintritt („fördert“). Die Vorschrift hat exakt die gleiche Zielrichtung wie der Art. 3 GG. Hinzu tritt, dass nach Art. 8 AEUV Gleichstellung nun eine Querschnittsaufgabe der Union ist: die Union wirkt bei allen ihren Tätigkeiten darauf hin, Ungleichheiten zu beseitigen und die Gleichstellung von Frauen und Männern zu fördern.

Der deutsche Gesetzgeber bleibt aufgefordert, zügig eine entsprechende Gesetzesinitiative zu ergreifen. Europäischer Mehrwert? Die weitere Modernisierung des Gesellschaftsrechts steht an.

Die Volkswirtschaft kann es sich nicht leisten, auf Frauen in den Chefetagen zu verzichten.

\footnotetext{
$20<$ <www.fidar.de> (Zugriff 13.7.2010).

21 Windbichler, Frauenquote für Aufsichtsräte (börsennotierter) Unternehmen, im Netz verfügbar unter <windbichler.rewi.hu-berlin. de/Frauenquote.pdf $>$ (Zugriff 13.7.2010), wovon Schladebach/Stefanopoulou, BB 2010, $1042 \mathrm{ff}$. aber offenbar ausgehen.
}

\title{
Wie heißt der alte Spruch? „Frauen und Männer sind gleichberechtigt - lieber gleich als später". Die Realität ist eher: Lieber später als gar nicht.
}

\section{Ingrid Claas \\ Rechtsanwältin, Wiesbaden}

Unter diesem Gesichtspunkt ist auch das Projekt zu sehen, das der djb beim „Frauen-Dinner Corporate Governance“ am 18. Oktober 2007 in Berlin auf den Weg gebracht hat. Gast des Abends war der damalige Vorsitzende der Regierungskommission Deutscher Corporate Governance Kodex (DCGK), Dr. Gerhard Cromme (Thyssen-Krupp). Ihm wurde eine Liste von 400 Frauen aus der Wirtschaft überreicht, die bereit und geeignet sind, einen Aufsichtsrat mit ihrem Fachwissen zu bereichern. Die Reaktion war, diplomatisch ausgedrückt, verhalten, was aber nicht so bleiben muss.

Die Bundesjustizministerin hat im September 2001 eine Regierungskommission eingesetzt, die am 26. Februar 2002 den Deutschen Corporate Governance Kodex verabschiedet hat. Er besitzt über die Entsprechenserklärung gemäß $\mathbb{} 161$ Aktiengesetz AktG eine gesetzliche Grundlage. Damit sollen die in Deutschland geltenden Regeln für Unternehmensleitung bzw. -überwachung für nationale und internationale Investoren transparenter gemacht werden. So soll das Vertrauen in die Unternehmensführung deutscher Gesellschaften gestärkt werden. Neuer Vorsitzender der Kommission ist seit Juli 2008 der Aufsichtsratsvorsitzende der Commerzbank AG, KlausPeter Müller. Zunächst in der Pressemitteilung vom 11. Februar 2010 und dann auch im Beschluss vom 26. Mai 2010 hat die Kommission als Arbeitsschwerpunkt für 2010 festgelegt: Besonders wichtig ist die Konkretisierung der Empfehlung für mehr Frauen und internationale Experten in Aufsichtsräten, das Vorbeugen von Interessenskonflikten in Aufsichtsräten, die Professionalisierung von Aufsichtsräten durch Fort- und Weiterbildung und der erste Bericht an die Bundesregierung im Herbst 2010. Diversity heißt Vielfalt. Die bisherige Diversity-Empfehlung ist für deutsche Aufsichtsräte mit dem Ziel zu konkretisieren, den Anteil von Frauen und internationalen Vertretern nachhaltig zu erhöhen. Dies soll in allen MDAXund DAX-30-Unternehmen vorgesehen werden. Weiter sind umfassende Fort- und Weiterbildungsmaßnahmen für künftige und amtierende Aufsichtsräte in vielfältiger Weise vorgesehen.

Würde die Quote wahr, müssten für ca. 750 Unternehmen bei jeweils nur zwei Frauen bereits 1500 Aufsichtsrätinnen gefunden werden - eine aufregende Zahl, die sich allerdings nicht von heute auf morgen verwirklichen lässt, sondern eher ein Projekt für die nächsten fünf bis zehn Jahre ist.

Das HV-Projekt 2010 ist, um auswertbar zu sein, klar strukturiert. Ziel ist, dass die djb-Frauen als Aktionärinnen oder Vertreterinnen von Aktionär(inn)en mindestens 70 Jahreshauptversammlungen von DAX-Unternehmen besuchen und beim Vorstand kritisch nachfragen, warum im Vorstand und im Aufsichtsrat keine oder, je nach Unternehmen, nur so wenige Frauen sind. Sind Frauen bereits im Aufsichtsrat eines Unternehmens, kommen sie in der Regel von der Arbeitnehmer(innen)seite, nicht jedoch von der Seite der Anteilseigner(innen). Hintergrund der Aktion ist, dass schriftliche Anfragen in der Vergangenheit in der Regel komplett ignoriert wurden. Nach dem Aktiengesetz ist der Vorstand allerdings verpflichtet, Fragen in der Hauptversammlung zu beantworten, wenn sie für 
die Tagesordnung relevant sind. Dies ist eine wunderbare Plattform, um Frauenthemen öffentlich zu machen.

Um es vorwegzunehmen: Bei den Antworten darf man keine Wunder erwarten. Obwohl die Fragen dem Vorstand meistens schon eine Woche vorher vorliegen und der Vorstand grundsätzlich die Möglichkeit hat, qualifiziert auf die Fragen zu antworten, ist dies in der Hauptversammlung nicht wirklich oder jedenfalls nicht immer zu erwarten. Bei meinem ersten Termin wussten die jungen Frauen am Eingang, dass der djb geschrieben hatte, was schon einmal ein sehr gutes Zeichen ist. Bis zum Vorstand war diese Information möglicherweise jedoch nicht vorgedrungen. Entsprechend waren die Antworten, die ich auf meine Fragen schließlich erhalten habe. Tatsächlich ist es mir nach einigem Drängen gelungen, schriftliche Antworten auf die Fragen zu erhalten. In der Sitzung selbst wurde der Aufsichtsrat bis zum Jahr 2014 neu gewählt. Das heißt: Die Termine für die Jahre 2011 bis 2014 sind schon fest eingeplant. Nur wenn das Unternehmen jedes Jahr gefragt wird, was sich seit dem letzten Jahr geändert hat und wie die Planung für 2014 aussieht, insbesondere im Hinblick auf die Aktivitäten des Nominierungsausschusses des Aufsichtsrates, kann langfristig eine Änderung der Unternehmenspolitik erreicht werden. Im Blick habe ich dabei die jungen Frauen im Unternehmen, die außerhalb des Protokolls vorsichtig mitgeteilt haben, dass für Verbesserungsmöglichkeiten im Unternehmen noch Raum sei und dass sie es gut finden, dass die Aktion durchgeführt wird.

Mein Wunsch ist zunächst eine medienwirksame Präsentationsveranstaltung im Herbst 2010 mit allen Aktivistinnen in Berlin, eventuell sogar zeitgleich, wenn der erste DCGB-Bericht der Bundesregierung übergeben wird.

Mein Wunsch ist es auch, dass allen Frauen, die an der Fortführung des Projektes interessiert sind, Fort- und Weiterbildungsmöglichkeiten angeboten werden, wenigstens ein Wochenendseminar zur Einstimmung, das die Arbeit im Aufsichtsrat unter frauenspezifischen Gesichtspunkten näher beleuchtet und insbesondere auch die immer wieder gestellte Frage nach der Qualifikation für eine solche Tätigkeit und inwieweit diese tatsächlich bei den Männern vorhanden ist.

Weiter ist es mein Wunsch, dass die Kolleginnen, die in diesem Jahr eine oder mehrere Jahreshauptversammlung(en) besucht haben, dies auch weiter tun, wenigstens bis zur nächsten Wahl der Aufsichtsräte, bzw. für eine Nachfolgerin sorgen, wenn sie selbst verhindert sind. Es ist auch sinnvoll, wenn der Besuch der Jahreshauptversammlung vorbereitet und nachbereitet wird, wenn nicht nur der Vorstand der AG die Fragen im Vorfeld erhält, sondern auch die Presse. Offensichtlich ist es auch erforderlich, Journalistinnen einen Tipp zu geben, damit sie zu den Jahreshauptversammlungen kommen und speziell über dieses Ereignis berichten. Anschließend sollte auch noch einmal ein Interview gegeben werden, in dem über die Bereitschaft des Vorstands, Fragen zu beantworten, berichtet wird. Für jeden Vorstand ist eine gute Pressemeldung wichtig. Wenn Antworten tatsächlich schriftlich nachgereicht werden, zwingt dies das Unternehmen zur Standortbestimmung und gibt eine
Basis für die kommenden Jahre. Daher ist es auch wichtig, Mittel für die nächsten Jahre beim Ministerium zu beantragen, damit die Aktion bis zur nächsten Wahl der Aufsichtsräte 2014 fortgesetzt werden kann. Ich halte es außerdem für sinnvoll, dass der Ingenieurinnenbund und der Journalistinnenbund mit ins Boot geholt werden. Es ist eine gute Möglichkeit, sich zu vernetzen, weil wir ein gemeinsames Ziel haben. Beide Organisationen können unser Projekt in wunderbarer Weise bereichern. Viele technische Unternehmen akzeptieren Juristinnen in Aufsichtsräten nicht, weil ihnen angeblich das nötige Knowhow fehlt, im Gegensatz zu Juristen im Aufsichtsrat, denen das Know-how üblicherweise nicht abgesprochen wird. Etwas Ähnliches kann von einer promovierten Ingenieurin nicht behauptet werden. Für Journalistinnen ergibt sich ein weites Arbeitsfeld, da sie vor und nach jeder Hauptversammlung mit Interviewwünschen an den Vorstand oder den Aufsichtsrat herantreten können, um über das Unternehmen zu schreiben und im Hinblick auf die Frauen in Führungspositionen Fakten zu sammeln. Unternehmenspolitik spielt sich nicht immer in der Öffentlichkeit ab. Die Jahreshauptversammlungen sind nur für Aktionär(inn)e(n) geöffnet. Daher ist es wichtig, dass Journalistinnen möglichst umfassend berichten.

Es ist die Ausnahme, dass die mündlich gestellten Fragen später noch einmal schriftlich beantwortet werden. Es gilt das gesprochene Wort und Aufzeichnungen sind, egal welcher Art, nicht erlaubt. Stenografieren ist dann die einzige Möglichkeit festzuhalten, was der Vorstand exakt gesagt hat. Bei Unternehmen, die diesbezüglich bereits aufgefallen sind, ist es sinnvoll, 2011 mit drei Aktionärinnen bzw. Aktionärsvertreterinnen in die Sitzung zu gehen, zwei, die die Fragen vortragen, und eine dritte, die die Antworten stenografiert. Ich glaube, dass der Vorstandsvorsitzende die Fragen gerne schriftlich beantworten wird, wenn ihm die Reinschrift seiner Antwort mit der Möglichkeit der Ergänzung präsentiert wird.

Mein Wunsch ist es, dass die angestellten Juristinnen und Betriebswirtschaftlerinnen der DAX-Unternehmen direkt angesprochen und eingeladen werden, Mitglied im djb zu werden. Es könnte vor Ort oder auch bundesweit eine Veranstaltung zum Thema Hauptversammlungen stattfinden, verbunden mit einer großen Mitgliederwerbeaktion. Über die Homepage können die Frauen, die im Unternehmen auf den Ebenen unter dem Vorstand sitzen, erreicht und ins Boot geholt werden. Spannend ist es auch, wenn Frauen, die die Jahreshauptversammlung besucht haben, und Frauen, die im Unternehmen in Führungspositionen sind, zusammenkommen und gemeinsam überlegen, was in den kommenden Jahren Erfolg versprechend ist.

Thema Quote: Es muss den Männern langsam super peinlich sein, nur über die 100-Prozent-Quote zu ihrem Job gekommen zu sein. Wir wollen die Zahl der Quotenmänner auf $60 \%$ reduzieren.

Der Weg ist noch lang. Ich sehe aber schon ein kleines Licht am Ende des Tunnels. Bei 750 Unternehmen, die erreicht werden sollen, lade ich alle ein, mitzutun und mitzugehen. 